\title{
A NOTE ON THE PERMANENTAL ROOTS OF BIPARTITE GRAPHS ${ }^{1}$
}

\author{
HePing Zhang, Shunyi LiU \\ AND \\ WEI LI \\ School of Mathematics and Statistics \\ Lanzhou University \\ Lanzhou, Gansu 730000, P.R. China \\ e-mail: zhanghp@lzu.edu.cn \\ liushuny@gmail.com \\ li_w07@lzu.edu.cn
}

\begin{abstract}
It is well-known that any graph has all real eigenvalues and a graph is bipartite if and only if its spectrum is symmetric with respect to the origin. We are interested in finding whether the permanental roots of a bipartite graph $G$ have symmetric property as the spectrum of $G$. In this note, we show that the permanental roots of bipartite graphs are symmetric with respect to the real and imaginary axes. Furthermore, we prove that any graph has no negative real permanental root, and any graph containing at least one edge has complex permanental roots.
\end{abstract}

Keywords: permanent, permanental polynomial, permanental roots.

2010 Mathematics Subject Classification: 05C31, 05C50, 15 A15.

\section{REFERENCES}

[1] B. Anderson, J. Jackson and M. Sitharam, Descartes' rule of signs revisited, Amer. Math. Monthly 105 (1998) 447-451. doi: $10.2307 / 3109807$

[2] F. Belardo, V.D. Filippis and S.K. Simić, Computing the permanental polynomial of matrix from a combinatorial viewpoint, MATCH Commun. Math. Comput. Chem. 66 (2011) 381-396.

\footnotetext{
${ }^{1}$ This work is supported by NSFC (grant Nos. 10831001, 61073046).
} 
[3] G.D. Birkhoff and D.C. Lewis, Chromatic polynomials, Trans. Amer. Math. Soc. 60 (1946) 355-451.

doi: $10.2307 / 1990348$

[4] M. Borowiecki, On spectrum and per-spectrum of graphs, Publ. Inst. Math. (Beograd) 38 (1985) 31-33.

[5] M. Borowiecki and T. Jóźwiak, A note on characteristic and permanental polynomials of multigraphs, in: Graph Theory, Borowiecki, Kennedy and Sysło (Ed(s)), (Berlin, Springer-Verlag, 1983) 75-78.

[6] M. Borowiecki and T. Jóźwiak, Computing the permanental polynomial of a multigraph, Discuss. Math. V (1982) 9-16.

[7] G.G. Cash, The permanental polynomial, J. Chem. Inf. Comput. Sci. 40 (2000) 1203-1206. doi:10.1021/ci000031d

[8] G.G. Cash, Permanental polynomials of smaller fullerenes, J. Chem. Inf. Comput. Sci. 40 (2000) 1207-1209. doi:10.1021/ci0000326

[9] R. Chen, A note on the relations between the permanental and characteristic polynomials of coronoid hydrocarbons, MATCH Commun. Math. Comput. Chem. 51 (2004) 137-148.

[10] D. Cvetković, M. Doob and H. Sachs, Spectra of Graphs: Third Edition (Johann Ambrosius Barth Verlag, Heidelberg-Leipzig, 1995).

[11] E.J. Farrell, An introduction to matching polynomials, J. Combin. Theory (B) 27 (1979) $75-86$. doi:10.1016/0095-8956(79)90070-4

[12] I. Gutman and G.G. Cash, Relations between the permanental and characteristic polynomials of fullerenes and benzenoid hydrocarbons, MATCH Commun. Math. Comput. Chem. 45 (2002) 55-70.

[13] D. Kasum, N. Trinajstić and I. Gutman, Chemical graph theory. III. On permanental polynomial, Croat. Chem. Acta 54 (1981) 321-328.

[14] L. Lovász and M.D. Plummer, Matching Theory, Annals of Discrete Mathematics, Vol. 29 (North-Holland, New York, 1986).

[15] R. Merris, K.R. Rebman and W. Watkins, Permanental polynomials of graphs, Linear Algebra Appl. 38 (1981) 273-288. doi:10.1016/0024-3795(81)90026-4

[16] J. Turner, Generalized matrix functions and the graph isomorphism problem, SIAM J. Appl. Math. 16 (1968) 520-526. doi:10.1137/0116041

[17] L.G. Valiant, The complexity of computing the permanent, Theoret. Comput. Sci. 8 (1979) 189-201. doi:10.1016/0304-3975(79)90044-6 
[18] W. Yan and F. Zhang, On the permanental polynomials of some graphs, J. Math. Chem. 35 (2004) 175-188.

doi:10.1023/B:JOMC.0000033254.54822.f8

[19] H. Zhang and W. Li, Computing the permanental polynomials of bipartite graphs by Pfaffian orientation, Discrete Appl. Math. 160 (2012) 2069-2074.

doi:10.1016/j.dam.2012.04.007

Received 18 June 2012

Revised 22 October 2012

Accepted 22 October 2012 